\title{
A Study on the "Length Approach to Speaking" English Teaching under the Hypothesis of Output
}

\author{
Ying Liu ${ }^{1}$ and Chen Huang ${ }^{2}$
}

Jingdezhen Ceramic University, Jingdezhen, Jiangxi 333403

\begin{abstract}
In recent years, the second language acquisition researchers have paid more and more attention to the cultivation of language output ability in English learning. Therefore, many language researchers have given some theoretical research and empirical evidence to improve the writing and speaking level of second language learners in study. Combined with the long teaching method and in order to improve the level of second language writing, Wang Chu Ming proposed "length approach to speaking" and get strong support. In this paper, under the guidance of Swain (1985) 's theory of language output hypothesis and the revelation of "writing long law", this paper explores the English teaching model of "the long law", and puts forward to improve students' English speaking and comprehensive application ability through a large number of lengthy English speaking training, i.e., to promote study with speaking. The author believes that "length approach to speaking" is feasible, operable and effective in the real language learning environment with security and low anxiety.
\end{abstract}

Keywords: Output hypothesis; Length approach to speaking; Teaching research

\section{输出假设下的英语 “说长法” 教学研究}

\author{
刘颖 ${ }^{1}$ 黄琛 $^{2}$ \\ （景德镇陶瓷大学，江西 景德镇333000） \\ 摘要: 近年来, 二语习得研究者越来越关注英语学习中语言输出能力的培养, 因此有不少的语言研究者为提高二语习得 \\ 者的写作和口语水平给出了一些理论研究和实证研究。结合教学法之长, 为提高二语写作水平, 王初明提出 “写长法” 并得 \\ 到有力的支持。该文在 Swain(1985)的语言输出假设理论的指导和 “写长法” 以写促学的启示下, 来探析 “说长法” 的英语 \\ 教学模式, 提出了通过 “说长法” 教学对学生进行大量的、长篇的英语口语输出训练来促进学生英语口语水平和英语综合应 \\ 用能力的提高, 即以说促学。笔者认为 “说长法” 在真实安全低焦虑的语言学习环境中也具有可行性、可操作性和有效性。 \\ 关键词: 输出假设; 说长法; 教学研究 \\ 中图分类号: G623.31 文摘标识码: A \\ 引言 \\ 长期以来困扰我国英语教学的一个难题是:虽然投入了大量的人力物力和时间, 学生的听、说、读、 \\ 写、译五大核心技能却未能得到均衡发展, 突出表现在其阅读能力应试能力较强, 口语水平和语言的实际 \\ 综合运用能力却非常欠缺。而与此同时在教学方法上却仍然存在着重输入轻输出的倾向, 教师着重讲解大 \\ 量的词汇、句法、阅读及应试技巧, 加上中国学生缺乏运用英语的情景环境, 输出的机会非常有限, 英语 \\ 教学的效果不容乐观, 令人焦虑。《大学英语课程教学要求》（2007）明确提出：“大学英语的教学目标 \\ 是培养学生的英语综合应用能力, 特别是听说能力, 使他们在今后工作和社会交往中能用英语有效地进行 \\ 口头和书面的信息交流。” 教学一线的英语教师非常有必要学习借鉴先进的教学理论和方法, 在实践中
}


不断运用和总结, 研究并发掘出英语口语突破的有效方法, 增强英语学习者综合运用英语的能力, 帮助他 们熟练地运用英语进行交流与沟通。

\section{1 “说长法” 教学的提出依据}

Krashen (1985) 提出输入假说，对第二语言的习得产生了重要影响。该假说认为，语言习得实现的条 件是学习者能够理解的语言输入 (comprehensible input); 语言输入包含稍高于其现有语言能力的语言项 目, 即著名的 “ $i+1$ ” 学说。

Swain (1995) 通过长期研究后发现: 只有大量的可理解输入而没有准确性的输出是不能保证学习者成 功习得二语的, 由此提出了 “输出假设” ( The Out-put Hypothesis )。学习者在语言交际方面达不到母 语水平的流利度、准确度和得体度, 原因之一是其缺少语言输出机会, 缺乏意义协商机会。Swain 研究的 结论认为口语、写作等输出对语言学习至少有四项功用: 提高表达的流利度, 增强学习者对语言点的敏感, 帮助学习者验证其关于语言的假设，有助于学习者反思、控制并进一步内化语言。

Skehan (1998) 指出语言输出还有另外两个功能:（1) 语言输出能培养学习者的话语能力; (2) 它还能树 立个人的话语风格 (personal voice), 建立自信心。

卢仁顺 (2002) 认为在外语习得实践中应重视语言输出作用，强化输出训练是解决我国英语界 “高分低 能, 哑巴英语, 费时低效” 的一个有效途径, 并且提出我国英语教学应该改变观念, 重视输出, 积极探索 强调语言输出作用的习得模式和测试方法。

“写长法”（王初明，2005）是以写的方式促进外语学习的方法。王初明指出, 这种方法以设计激发 写作冲动的任务为教学重点, 在一定的学习阶段, 顺应外语学习规律, 通过调节作文长度要求, 逐步加大 写作量, 使学生在表达真情实感的过程中, 打破外语学习的极限, 以 “写长” 体验去克服外语学习的心理 障碍, 以激发创作成就感来释放学习潜力, 以写促学。由此增强学习成就感, 提高信心, 将外语知识加速 打造成外语运用能力。“写长法” 是对输出假设理论和二语习得理论研究的结晶, 英语教育界知名学者桂 诗春、钱冠连、何自然、王立非、李力等对 “写长法” 取得的成绩予以了充分的肯定。

语言输出包括说和写两大技能，在首届全国 “以写促学” 写作会议上 (2003，广州)，钱冠连提出过 “说 长法” 模式的设想，并且举例说明了它的可行性。“说长法” 正是基于 “写长法” 的启示拓展出的一种 英语口语教学法, 其基本理念与 “写长法”一脉相承, 即: 说长, 多说。教学核心旨在通过设计激发学生 兴趣的任务, 在学生充分准备的情况下让学生有话可说, 主动去说, 愿意去说, 延长口语输出的时长, 扩 充口语输出的内容。对学生进行大量的, 长篇的英语口语输出训练, 能显著地提高学生的英语口语水平, 降低开口焦虑, 提高学生的英语综合应用能力。

\section{2 “说长法” 教学的可行性}

“说长法” 教学实践以 “说” 为突破口, 符合英语语言学习规律, 其内在的交际二语习得理念即语言 是用于交际的。通过大量的 “读” 和 “听” 的语言输入可以促使学生的语感的形成和口语流利程度的提高, 在已有的 “语言输入” 的基础上, 以大量的 “语言输出” 行为进行教学活动, 才能真正全面提高学生运用 英语的能力。语言输出能力的习得顺序为先说后写, 以量促质, 以说促学可以有效促进语言知识的内在联 系, 符合认知心理规律。 
改革开放以来, 随着我国与世界各国的交往更加频繁和深入, 英语口语表达能力的重要性也日益突出。 英语作为一种交流的工具, 在我国的社会经济发展中起着至关重要的作用, 说与写的能力是学生英语交际 能力最重要的体现, 更是培养学生交际能力的必要手段和目标。“说长法” 顺应我国学生英语学习的迫切 需求，改善长期困扰我国学生 “哑巴英语” 的现状。

“说长法” 教学一方面可以改善传统教学中以教师为中心的教学定位和以教师的灌输和教授为主导 的学习方法和学习观; 另一方面, 在教与学的过程中可以设计和营造更真实、更贴近生活、学习者更能接 受和融入的目的语环境, 最大程度激发学习者的学习兴趣和热情, 帮助他们建立新旧知识的有效连结, 提 高语言学习效率。在高校英语课程中实施 “说长法” 能有效引导学习观念朝着更有利于英语学习的方向转 变, 学习者在 “说长法” 课堂聚焦口语输出能力的同时附带获得学习能力的综合提高。

\section{3 输出假设理论下的 “说长法” 的教学设计与操作}

语言输出能力是英语教学的终极目标, 说与写的能力是语言能力的最佳反映。针对学生英语学习多年 依然无法 “活学活用, 学以致用” 的状况, “说长法” 以激发学生在轻松自在的课堂环境中表达真情实感 和完成输出口语任务为教学重点, 通过研究英语的学习规律来调节任务难度、情景设置的难度、丰富教学 形式和课堂资源。从而促使学生摆脱口头输出时的思想包袱, 降低口语焦虑, 走出哑巴英语的困境, 由此 增强学习成就感提高自信心将英语知识转变成英语运用能力。

“说长法” 教学可分为课堂内外两大板块。课外教师应根据学生已有的英语基础和对语言技能的应用 情况, 紧密围绕教学主题和大纲要求, 精心设置恰当的情境设计恰当的口语任务提出恰当的问题。情境的 设置要遵从学生对所学内容的理解, 需要反映学生日常生活中复杂的社会环境。另外教师应设定真实的任 务, 以此激发学生联系实际生活的能力, 鼓励他们在社会生活背景中解决问题。问题的类型分开放型和封 闭型, 开放型的问题能为学生提供更大的想象空间, 调动学生的多元智能。教师将情境设定, 口语任务及 对说长时间的要求明确告知学生并吸引和启发学生进入问题情境中, 慢慢放手让学生独立去寻求问题的答 案和任务的解决方法。

学生为提高课内口语输出任务完成的质量主动进行语音学习和纠音练习, 大量跟读模仿标准地道的音 视频，还可以进行朗读背诵训练。根据口语任务的主题，学生提前利用各种信息资源，互联网图书馆等方 式检索相关的文化背景资料，并阅读各类英文原版报纸期刊、新闻视听材料等，熟悉积累相关词汇，扩大 知识面。也可小组合作，互相分享交流讨论，综合归纳自我观点和同伴经验为课内能够高效完成口语输出 做好前期准备工作。

课内教师调整自我定位, 为学生创造安全轻松低焦虑的学习环境的前提下, 在学生学习过程中充当引 导者、支持者和指导者的角色。首先教师应吸引和引导学生融入既定的教学情境使学生理解特定的教学任 务, 可借助影视音乐作品导入唤醒学生的多元智能。鼓励学生在协作教学活动中积极参与, 踊跃表现, 发 挥作用, 完成小组任务, 从而进一步实现语言的内化。其次教师要帮助学生确定目标、寻求挑战、认识变 化、引导学生发现问题发现学习规律从而加深对学习内容的理解。最后教师还要为学生创造良好的学习心 理环境帮助学生发展自我控制学习行为的意识和能力增强自信心。

说长法教学中应把个体输出和小组协作相互结合。让学生在小组互动中学习讨论、协商把原本矛盾的 观点逐渐统一起来, 形成新的观点, 最终完成对所学知识的深入思考和理解。在做练习时教师可以安排学 
生互评, 也可让学生把口语输出任务录下来, 以录音的方式互评, 更能降低开口焦虑。他们在互评过程中 发现自身和同学的错误, 学会分析错误, 总结错误。通过互评促进学生之间相互学习的兴趣和热情, 取长 补短, 以达到提高学生口语输出的兴趣和能力。这样既能改变学生的自我学习观念, 又能增强学生的自信 心和成就感。

对活动形式设计、教学内容的规划、情景的设置、教学任务的安排、协作教学形式及内容的处理以及 学习资源的使用等环节的设计应严格遵从从易到难的规律。这是 “说长法” 的核点和灵魂, 也是关系着是 否可以成功 “以说促学” 的关键。

\section{4 结语}

英语犹如人的第三只眼, 在全球化飞速发展的时代最大化发挥英语作用以接触更广阔的世界是一项重 要议题。如何结合我国大学英语教学实际, 切实提高学生的英语应用能力是教师面对的首要任务。本文提 出的 “说长法” 是建立在语言的本质、英语学习的特点以及英语课堂的根本作用的基础上, 旨在通过在既 定的 “情景” 下学生借助 “说” 和完成 “既定任务” 的形式来提高学生综合的语言运用能力和交际能力。 英语口语教学是一项复杂、系统的工程随着学习对象、环境、方法等的改变而产生不同的学习效果。我们 只有本着因人制宜、因时制宜、因事制宜、因任务制宜、因情景制宜的原则，灵活多变地设计课堂教学才 能最大程度发挥“说长法”的有效性和可操作性。

\section{5 致谢}

基金项目：景德镇陶瓷大学校自选社会科学研究项目

\section{Acknowledgement}

Fund Project: Selected Social Science Research Project of Jingdezhen Ceramic University

\section{参考文献}

[1] Krashen S. The Input Hypothesis: Issues and Implications[M] . London: Longman,1985.

[2] Swain, M. Communicative competence: Some roles of comprehensible input and comprehensible output in its development [A]. S, Gas. and C. Madden, Input in Second Language Acquisition [C]. Rowley, MA: Newbury House,1985.

[3] Swain, M. Three Functions of Output in Second Language Learning [M]. Shanghai: Shanghai Foreign Language Education Press, 1995.

[4] 王初明, 牛瑞英, 郑小湘。以写促学[J]。外语教学与研究, 2000（3）:207-212.

[5] 卢仁顺。“输出假设”研究对我国英语教学的启示[J]。外语与外语教学，2002，(4) : 34-37.

作者简介：

黄琛（1985-）女，硕士，景德镇陶瓷大学讲师，研究方向为英语语言文学

刘颖 (1985-) 女，硕士，景德镇陶瓷大学助教，研究方向为二语习得及英语教学

\section{References:}

[1] Krashen S. The Input Hypothesis: Issues and Implications [M]. London: Longman, 1985.

[2] Swain, M. Communicative competence: Some roles of comprehensible input and comprehensible output in its development [A]. S, Gas. And C. Madden, Input in Second Language Acquisition [C]. Rowley, MA: Newbury House, 1985.

[3] Swain, M. Three Functions of Output in Second Language Learning [M]. Shanghai: Shanghai Foreign Language Education Press, 1995. 
[4] Wang Chuming, Niu Ruiying, Zheng Xiaoxiang. Promoting Learning with Writing [J]. Foreign Language Teaching and Research, 2000(3):207-212.

[5] Lu Renshun. The Enlightenment of "Output Hypothesis" Research on English Teaching in China [J]. Foreign Language Teaching and Research, 2002, (4) :34-37. 